Utah State University

DigitalCommons@USU

Electrical and Computer Engineering Student

Research

Electrical and Computer Engineering Student

Works

$12-1-2016$

\title{
Adaptive Biasing Cell Association in FFR Aided Multi-tier Heterogeneous Networks under Dynamic Load Variation
}

\author{
Didarul Alam \\ Khulna University of Engineering \& Technology (KUET) \\ Tashikur Rahman \\ Khulna University of Engineering \& Technology (KUET) \\ Shakil Ahmed \\ Utah State University, shakil.ahmed@aggiemail.usu.edu \\ Mostafa Zaman Chowdhury \\ Khulna University of Engineering \& Technology (KUET)
}

Follow this and additional works at: https://digitalcommons.usu.edu/ece_stures

Part of the Electrical and Computer Engineering Commons

\section{Recommended Citation \\ Md. Tashikur Rahman, Md. Didarul Alam, Shakil Ahmed, and Mostafa Zaman Chowdhury "Adaptive Biasing Cell Association in FFR Aided Multi-tier Heterogeneous Networks under Dynamic Load Variation" In Proceeding 5th IEEE International Conference on Informatics, Electronics and Vision (ICIEV), May 2016, pp. 829-833.}

This Conference Paper is brought to you for free and open access by the Electrical and Computer Engineering Student Works at DigitalCommons@USU. It has been accepted for inclusion in Electrical and Computer Engineering Student Research by an authorized administrator of DigitalCommons@USU. For more information, please contact digitalcommons@usu.edu.

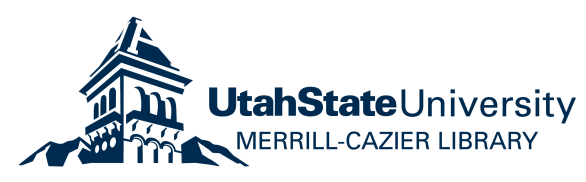




\title{
Adaptive Biasing Cell Association in FFR Aided Multi-tier Heterogeneous Networks under Dynamic Load Variation
}

\author{
Md. Didarul Alam, Md. Tashikur Rahman, Shakil Ahmed, and Mostafa Zaman Chowdhury \\ Dept. of Electrical and Electronic Engineering, Khulna University of Engineering \& Technology, Bangladesh \\ E-mail: didar_2k11@hotmail.com, rahmantashik@gmail.com, shakileee076@gmail.com, mzceee@yahoo.com.
}

\begin{abstract}
Heterogeneous networks (HetNets) adopting fractional frequency reuse (FFR) improves cell coverage, network capacity, efficiency, assures higher data rates, and better quality of service $(\mathrm{QoS})$ for next generation wireless networks. However they fail to handle dynamic load variation. So we attempt biasing cell association (BCA) to offload user from macrocell to small cell base stations (SCBs) to overcome capacity reduction and throughput degradation. It is based on range expansion of SCBSs by adding a positive bias to the reference signal received power (RSRP). In this paper we propose a FFR aided twin layer HetNet with an adaptive biasing scheme for load balancing. For users offloading a cell is selected by self-organizing network (SON) with adaptive bias value using $Q$-learning algorithm. Simulation result show that our system model can handle dynamic load variation with proper utilization of available bandwidth and mitigate interference better than the conventional HetNet design.
\end{abstract}

Keywords - HetNets, FFR, QoS, BCA, SON, Q-learning.

\section{INTRODUCTION}

Future wireless networks are facing explosive data demands with the proliferation of various multimedia traffic types. Heterogeneous networks (HetNets) consisting of macrocells overlaid with small cells (femtocells, picocells) is one of the most promising technology to meet the guaranteed level of quality of service (QoS). For the drastic increase of traffic it is necessary to ensure maximum utilization of radio resource with using the same frequencies repeatedly across a cellular system with little potential for interference. Authors in [1] have allocated radio resource between cell centre (CC) and cell edge (CE) region to ensure fairness. However, distribution of users are hardly uniform in a cell coverage area. So spectrum allocation in different region of cell coverage area are not optimal under dynamic load variation. Authors in [2] have proposed a dynamic channel borrowing scheme for reused frequency allocation between neighbouring macrocells. In [3] authors analysed to offload user to small cell base stations (SCBSs) by adding a fixed range expansion bias, however a fixed bias value can't always provide a good performance in changing environment for different traffic scenarios. Authors in [4] have addressed the issue of load aware cell selection without considering inter-cell interference mitigation techniques. Authors in [5] proposed Q-learning for cell selection to offload users. Selection of optimal bias value for maximising throughput is also a key issue under dynamic load variation.
Offloading users from macrocell base station (MBS) to $\mathrm{SCBs}$ can be controlled via manipulating relative transmission power of SCBs, relative intensity of SCBs and biasing factor [6]. Transmission power control is a method for range extension and load balancing [11]. User offload from overloaded MBS to lightly loaded SCBs reduce the overloaded traffic served by the MBS to enhance their QoS and increase the overall system capacity with decreasing call blocking probability.

Biasing cell association (BCA) is an effective approach for load balancing technology in HetNets. BCA offloads users from MBS to SCBs by adding a positive bias to the reference signal received power (RSRP) [7]. By applying bias, a SCBs can attract more users expanding the cell association region. Fig. 3 shows BCA concept for selection of SCBs over MBS. The dotted line in Fig. 3 shows the additional coverage area of SCBs. The main problem of BCA is the interference for using same frequency bands between macrocells and femtocells. We propose a two-tier HetNets with fractional frequency reuse (FFR) for proper partitioning and allocating different frequency bands for different users of MBS and FBS to eliminate intercell interference and under heavy load traffic offload users from MBS to FBS to optimize the overall experience of users. The proposed biasing scheme provides SCBs with optimum bias value for load balancing by a learning algorithm in changing environment.

For proper cell selection we suggest SON which main functions are self-configuration (frequency planning and deployment), self-optimization (neighbouring list optimization, coverage and capacity optimization) and self-healing (automatic detection and solution, cell outage compensation) [9]. We proposed a mechanism to create a neighbour cell list with a minimum, but appropriate number of cells for offloading users.

Q-learning is the optimal action-selection policy learning algorithm. To avoid imbalance of traffic amount, we proposed Q-learning to select appropriate bias value for range expansion. The steps are initialize $\mathrm{Q}$ table, take an action corresponding the current state, observe the outcome state, collect the reward function and finally update the Q-table [5].

The rest of this paper is organized as follows. Section II shows the proposed model with frequency planning and BCA for user offload. In Section III fair resource allocation with capacity and outage probability analysis of proposed scheme is shown. Performance Evaluation and concluding notes are drawn in Section IV and Section V. 


\section{PROPOSED MODEL}

In our proposed model we consider a cluster of 3 cells, each cell using FFR-3 [8] (frequency reuse factor one for CC user and reuse factor 3 for $\mathrm{CE}$ users). The sub-carrier allocation is based on orthogonal frequency-division multiple access (OFDMA) cellular system. So that intra-cell interference can be mitigated. Figure 2 (a) shows interference scenario when MBS overlaid with femtocell base station (FBS). We consider femtocells as SCBs. Figure 2 (b) shows the FFR-3 frequency planning for a single cell. Here $\mathrm{R}$ represents the total macrocell coverage radius, $\alpha$ is the ratio of $\mathrm{CC}$ region, $\beta$ is the bandwidth factor allocated for $\mathrm{CC}$ users and $\mathrm{W}$ is the total bandwidth. Our system model involves the selection of proper values of $\alpha$ and $\beta$ for non-uniform user distribution so that the resource allocation for existing number of users experience acceptable per user capacity. Under heavy traffic load, setting up adaptive bias value for FBS to offload macrocell users onto FBS. Optimal bias factor should be selected as higher biasing value will extend the range of femtocell beyond its optimal range. As FBS can only accommodate few users, beyond that additional user will reduce total throughput. Introduction of FFR-3 allocates 3 different frequency bands. So the FBSs can choose one from three. Without FFR-3 a FBS uses same frequency as the MBS, so femtocell users are affected by large inter-channel interference. In this model FBS operates in open access, which mean the users are permitted to access any FBS so that macrocell user equipment (MUE) can be handed over to it. Fig. 1 shows the interference suffered by a user equipment (UE) associated with a FBS if neighbouring FBSs use same frequency. With FFR-3 neighbouring FBSs have different frequency, so UE gets an interference free signal reception. To find the target femtocell access point (FAP) for user offloading, we suggest self-organizing network (SON). Under heavy traffic mobile station (MS) detects signal from femtocell and sends measurement report to the connected MBS. Location information is exchanged among the neighbour FBSs as well as MBS and finally the combination of the MS, MBS and neighbouring FBSs performing SON configuration to create an optimized neighbouring cell list for load balancing. On the basis of pre-authenticated and received signal levels the MS selects the target femto access point (FAP) [9]. For bias value selection we propose Q-learning algorithm, where an agent tries to learn the optimal bias value from its history of interaction with the environment.

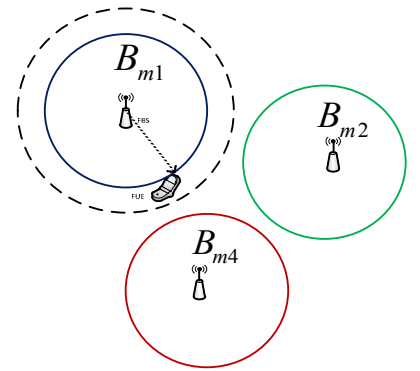

(a)

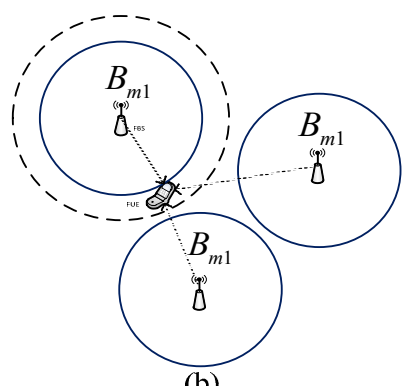

(b)
Figure 1 Frequency band allocation between FBS (a) with FFR-3 and (b) without FFR.

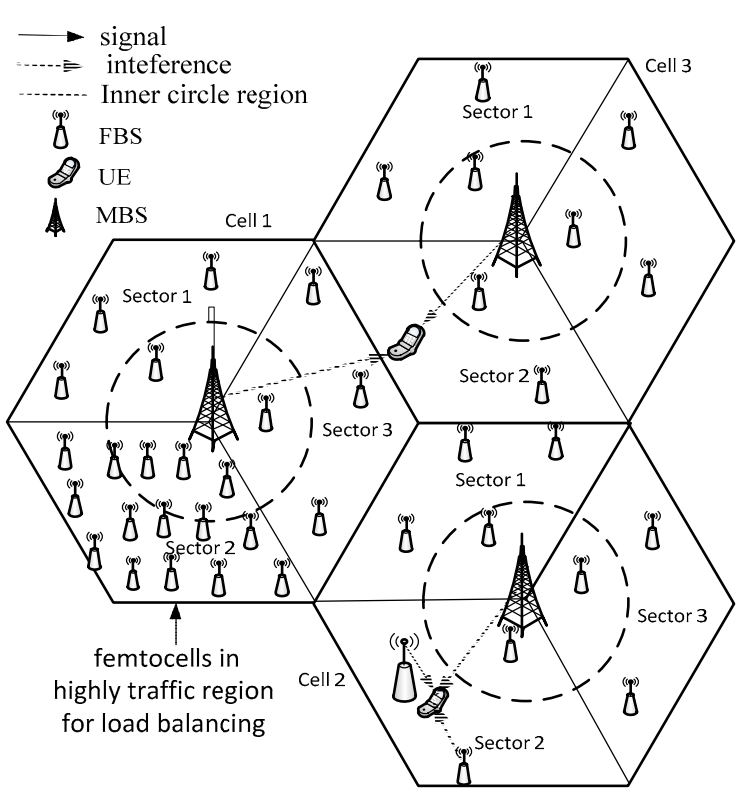

(a)

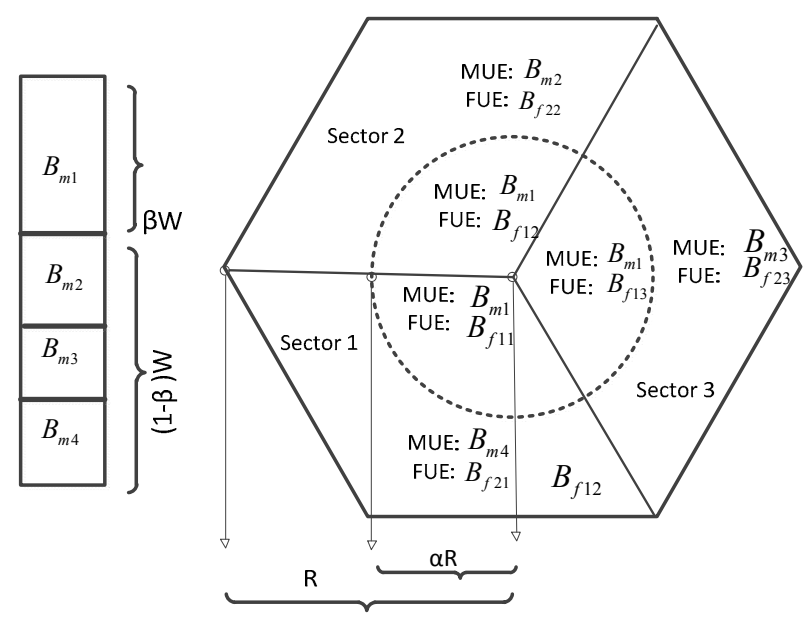

(b)

Figure 2 Heterogeneous network design (a) A cluster of three macrocell overlaid with femtocells (b) Single cell with FFR-3 frequency planning.

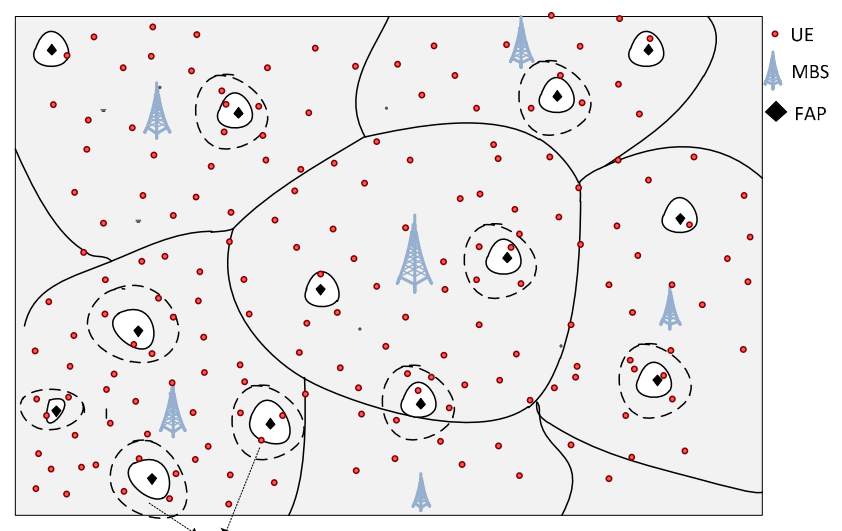

Cell rangr expansion

Figure 3 A two-tier HetNet model with BCA scheme for user offload to SCBSs (femtocells). 


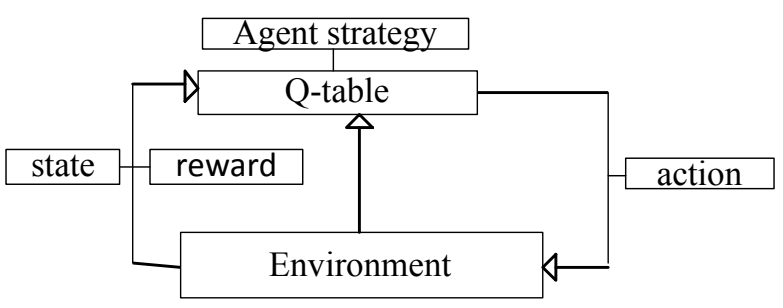

Figure 4 The flow of Q-learning

The state-action-reward function is

$$
\{\mathrm{S}, \mathrm{A}, \mathrm{R}, \mathrm{S}\}=\text { agent-state, action, reward,new-state }\}
$$

In Figure 4, a pilot signal acts as an agent, optimized bias value is selected as the action, the QoS, per user capacity, outage probability, backhaul resource utilization is considered as the reward function. In each step pilot signal explores the environment, receive the reward function until it reaches the goal state and selecting the optimum bias value.

For frequency utilization between MBS an FBS, the following set of equations can be written

$$
\begin{aligned}
& \left|B_{T}\right|=\left|B_{m 1}\right|+\left|B_{m 2}\right|+\left|B_{m 3}\right|+\left|B_{m 4}\right| \\
& \left|B_{m 1}\right|=\beta W \\
& \left|B_{m 2}\right|=\left|B_{m 3}\right|=\left|B_{m 4}\right|=\frac{(1-\beta) \mathrm{W}}{3} \\
& \left|B_{f 11}\right|=\left|B_{m 2}\right|+\left|B_{m 3}\right| \\
& \left|B_{f 12}\right|=\left|B_{m 3}\right|+\left|B_{m 4}\right| \\
& \left|B_{f 13}\right|=\left|B_{m 2}\right|+\left|B_{m 4}\right| \\
& \left|B_{f 21}\right|=\left|B_{m 1}\right|+\left|B_{m 2}\right|+\left|B_{m 3}\right| \\
& \left|B_{f 22}\right|=\left|B_{m 1}\right|+\left|B_{m 3}\right|+\left|B_{m 4}\right| \\
& \left|B_{f 23}\right|=\left|B_{m 1}\right|+\left|B_{m 2}\right|+\left|B_{m 4}\right| \\
& \left|B_{m 1}\right| \cup\left|B_{f 11}\right| \cup\left|B_{f 21}\right|=\left|B_{T}\right| \\
& \left|B_{m 2}\right| \cup\left|B_{f 12}\right| \cup\left|B_{f 22}\right|=\left|B_{T}\right| \\
& \left|B_{m 3}\right| \cup\left|B_{f 13}\right| \cup\left|B_{f 23}\right|=\left|B_{T}\right|
\end{aligned}
$$

where $B_{f m n}$ represents bandwidth allocation of femtocell. $\mathrm{m}=1$ represent $\mathrm{CC}$ region, $\mathrm{m}=2$ represents $\mathrm{CE}$ region. $\mathrm{n}$ represents the sector number. $B_{m 1}, B_{m 2}, B_{m 3}, B_{m 4}$ are the sub band of $B_{T}$.

Algorithm 1: mechanism for cell selection and adaptive bias value configure to offload user

1: While a call arrives at reference cells (cell 1) do

2: If user is uniformly distributed in MBS then

3: Allocate bandwidth with varying radius to ensure maximum per user capacity

$$
\text { end if }
$$

while the macrocell is overloaded do

perform SON to create neighbouring femtocells list

if $R S R P_{i, \text { femto }}>R S R P_{\mathrm{j}, \text { femto }}$

MS select $i^{\text {th }}$ femtocell as its target AP

else
MS select $j^{\text {th }}$ femtocell as its target AP

end if

selection of proper bias values by Q learning algorithm let $\mathrm{t}=0$,

measures throughput, resource utilization and outage for state

for state $S_{t}^{i}=\left\{\mathrm{s}_{t, 1}, \mathrm{~s}_{t, 2} \ldots \ldots \mathrm{s}_{t, \mathrm{k}}\right\}$

Action $A_{t}^{i}=\left\{\mathrm{B}_{t, 1}, \mathrm{~B}_{t, 2} \ldots \ldots \mathrm{B}_{t, \mathrm{k}}\right\}$

reward $R_{t}^{i}=\left\{\mathrm{r}_{t, 1}, \mathrm{r}_{t, 2} \ldots . . \mathrm{r}_{t, \mathrm{k}}\right\}$

Q value $Q\left(\mathrm{~S}_{t}{ }^{i}, \mathrm{~A}_{t}{ }^{i}\right)$

end for

while the BS evaluates its current state $S_{t}^{i}$

if the state is not changed then

exit

else

generate a random number y $(0 \leq y \leq 1)$

if $y<\in(\in$-greedy policy, $(0 \leq \in \leq 1))$ then

select a bias value randomly

else

select the bias value with the maximum Q-value

end if

end if

send an access request to the target AP

calculate the reward $r_{i, t+1}=\left(\Theta_{t, k}, C_{t, u e}\right)$

update $\mathrm{Q}$ table according to

$Q\left(\mathrm{~s}_{t}, a_{t}\right) \leftarrow(1-\ell) Q\left(\mathrm{~s}_{t}, a_{t}\right)+\ell\left[\mathrm{r}_{t+1}+\partial \max _{a \in A_{i}} \mathrm{Q}\left(\mathrm{s}_{t+1}, \mathrm{a}\right)\right]$

learning rate $0<\ell \leq 1$, discount factor $0<\partial \leq 1$

end while

end while

end while

\section{PERFORMANCE EVALUATION}

The performance analysis of our proposed scheme will be done based on adaptive BCA with SINR level and outage probability. We consider one macrocell which consists of MBS and several FBS. The capacity achieved by users of both MBS and FBS is a prime factor. Okumura-Hata model is used in literature [2]. According to this the propagation model for macrocell case can be expressed as

$$
\begin{aligned}
& L=36.55+26.16 \log _{10} f_{c, m}-3.82 \log _{10}\left(h_{b}\right)-a\left(h_{m}\right) \\
& +\left[44.9-6.55 \log _{10} h_{b}\right] \log _{10} d+L_{s h}[\mathrm{~dB}] \\
& a\left(h_{m}\right)=1.1\left[\log _{10} f_{c, m}-0.7\right] h_{m}-\left(1.56 \log _{10} f_{c, m}-.8\right)
\end{aligned}
$$

where $\mathrm{L}$ is the path loss exponent for cellular networks, $f_{c, m}$ is the centre frequency of the macrocell in $\mathrm{MHz}, h_{m}$ and $h_{b}$ is the height of the MBS and FBS in meter respectively, $d$ is the distance between the MBS and the MS in kilometre, $L_{s h}$ is the shadowing standard deviation. The propagation model for a femtocell can be expressed

$$
L_{\text {femto }}=20 \log _{10} f_{c, f}+N \log _{10} d_{1}-28[\mathrm{~dB}]
$$

where $f_{c, f}$ is the centre frequency of the femtocell in $\mathrm{MHz}, d_{1}$ is the distance between The FBS and the MS in meter.

The received SINR of a typical user associated with a MBS or FBS integrated network can be expressed as: 


$$
\gamma_{k}(\mathrm{x})=\frac{P_{k} * 10^{-\frac{L_{p, k}(\mathrm{x})}{10}}}{\sum_{i=0}^{M} I_{m(i)}+\sum_{j=1}^{X} I_{n(j)}+N}
$$

where the transmitted power of BS (MBS/FBS) is $P_{k \in Z}, L_{p, k \in Z}(\mathrm{x})$ is the Path loss , $\mathrm{N}$ is the noise generated by various sources. $\mathrm{Z}$ is the total number of APs. $I_{m(i)}$ Is the power of the interference signal from the $i^{\text {th }}$ interfering macrocell from among the $M$ interfering macrocells, and $I_{n(j)}$ is the received interference signal from the $j^{\text {th }}$ femtocell from among the $X$ neighbouring femtocells. The $i^{\text {th }} \mathrm{UE}$ will select the $k^{\text {th }} \mathrm{AP}$ as its serving BS if [7],

$$
\begin{aligned}
& \text { CellD }_{k}=\arg \max _{k}\left(\mathrm{RSRP}_{k}\right) \\
& \text { CellD serving }=\arg \max _{k}\left(\mathrm{RSRP}_{k}+\mathrm{B}_{k}\right)
\end{aligned}
$$

$\mathrm{B}_{k}$ is the biasing factor of BS $\mathrm{k}$. Generally set $\mathrm{B}_{k}=0$ for all MBS and $\mathrm{B}_{k}>0$ for FBS. Because our aim is to virtually expand the femtocells range by adding some positive value not expand the macrocell range. $\mathrm{B}_{k}$ is set between $\mathrm{B}_{\min }<\mathrm{B}_{k}<\mathrm{B}_{\max }$. Authors in [7], [10] suggest that the $\mathrm{B}_{\min }=0 d B$ and $\mathrm{B}_{\max }=20 \mathrm{~dB}$. If the biasing factor will low the SCBSs (femtocells) will unable to attract most of the users, so the resources won't be fully exploited. On the other hand if the biasing factor will high, many user will offload to SCBSs (femtocells) and the larger distance UE will experience bad RSRP. Per user capacity at CC and $\mathrm{CE}$ region is expressed as

$$
\begin{gathered}
C_{u, c}=\frac{\beta W R^{2}}{2 N_{u, c} \int_{0}^{\alpha R} 1 / \log _{2}(1+\gamma)} \\
C_{u, e}=\frac{(1-\beta) W R^{2}}{2 N_{u, e} \int_{\alpha,}^{R} 1 / \log _{2}(1+\gamma)}
\end{gathered}
$$

where $N_{u, c}$ and $N_{u, e}$ is the total number of user in CC and CE region. The Shannon Capacity Formula

$$
C=W \log _{2}(1+\gamma)[\text { bits } / s]
$$

The outage probability of a user is calculated as:

$$
\Theta_{k}=P_{r}\left(\gamma_{k} \leq \zeta\right)
$$

where $\zeta$ is the threshold value of $\operatorname{SINR}$ below which there is no acceptable signal reception. Considering all the interfering neighbour macrocells and femtocells, the outage probability can be expressed as

$$
\mathrm{O}_{k}=1-e^{(-\zeta / \gamma)}
$$

\section{SIMULATION RESULTS}

In this section we analyse the performance of our proposed scheme in terms of available per user capacity of MUE for both $\mathrm{CC}$ and $\mathrm{CE}$ users, spectral efficiency and outage probability of FUE comparing with without FFR and FFR-3 with different bias value. Table I shows the parameter values used in our analysis. Fig. 5 shows the selection of optimal values of radius factor $(\alpha)$ and bandwidth factor $(\beta)$ for maximise capacity of $\mathrm{CE}$ users within the cell. Here 500 users have been considered. In Fig. 5 as the bandwidth factor decreases with increasing radius factor per user capacity decreases. On the other hand, for $\mathrm{CC}$ users as the bandwidth factor increases with decreasing radius factor per user capacity increases. So the CE and CC user's capacity can be optimal by proper selection of radius factor with bandwidth factor. In analysis the distance of users are also varying. However as the distance between the UE and MBS increases SINR value is decreases, so capacity decreases. CE users experience worst bit rate due to larger path loss. In our performance analysis, due to low transmitted power and large path loss only those femtocells within 60 meter range of reference femtocell are regarded as potential source of interference.

TABLE I Summary of the simulation parameters

\begin{tabular}{|l|l|}
\hline Parameter & Value \\
\hline Radius of macrocell & $500(\mathrm{~m})$ \\
\hline Radius of femtocell & $10(\mathrm{~m})$ \\
\hline MBS transmit power & $1.5(\mathrm{~kW})$ \\
\hline FBS transmit power & $10 \mathrm{~mW}$ \\
\hline Channel bandwidth & $4.8 \mathrm{MHz}$ \\
\hline Carrier frequency & $900(\mathrm{MHz})$ \\
\hline Bandwidth factor $\beta$ & $0.01<\beta<1$ \\
\hline Radius factor $\alpha$ & $0.01<\alpha<1$ \\
\hline Macro base station height & $50(\mathrm{~m})$ \\
\hline Femto base station height & $2(\mathrm{~m})$ \\
\hline$\zeta$ & $7(\mathrm{~dB})$ \\
\hline
\end{tabular}

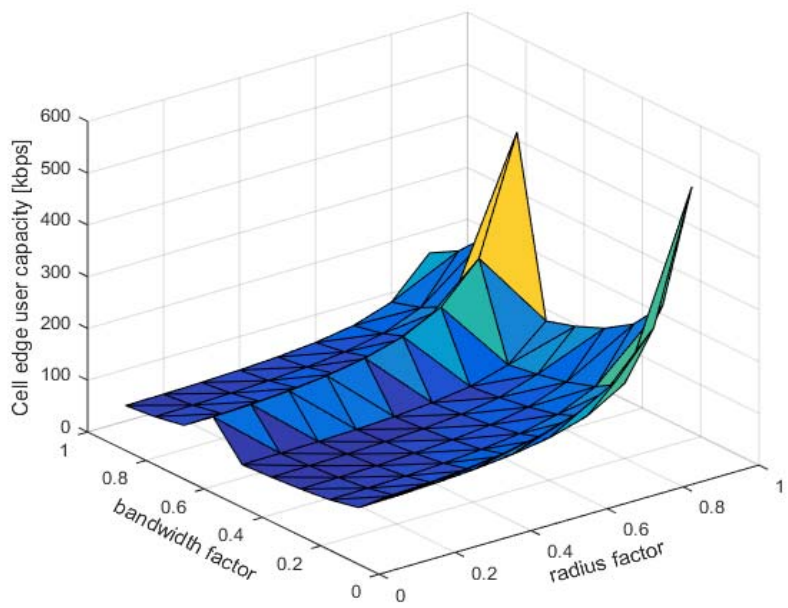

Figure 5 Selection of $\alpha$ and $\beta$ for maximum per user capacity at CE. 


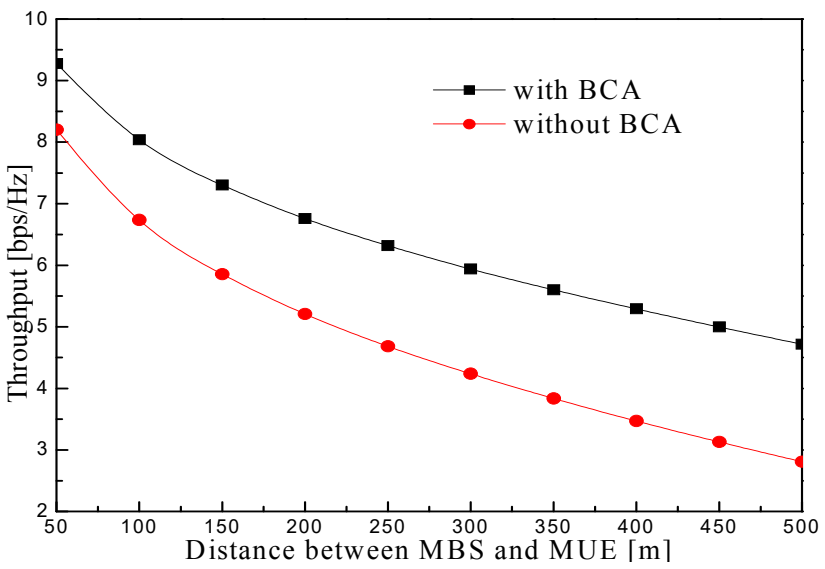

Figure 6 Throughput comparison for MUEs with respect to distance from MBS.

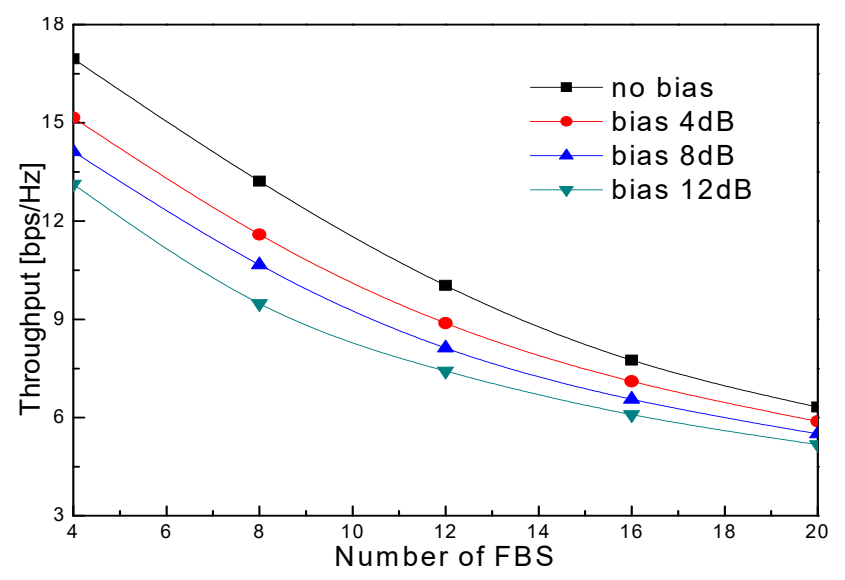

Figure 7 Throughput comparison for FUE with BCA.

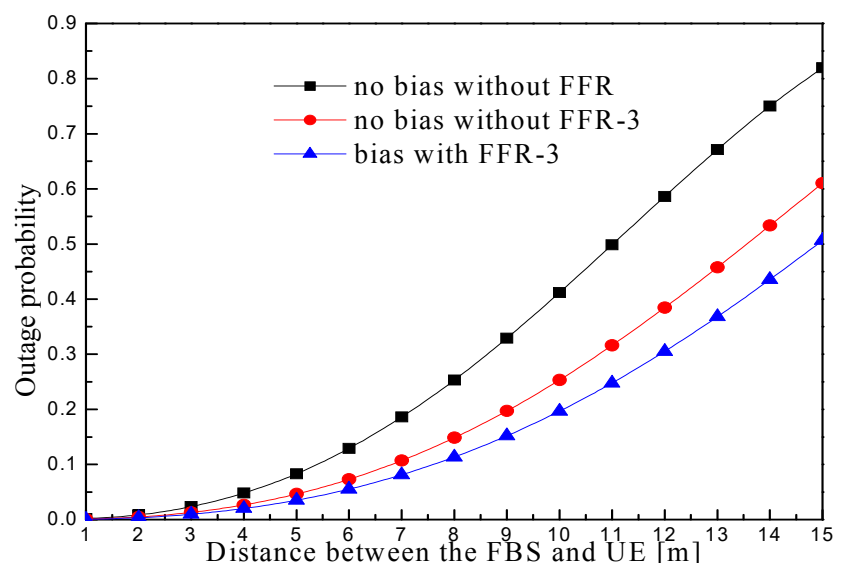

Figure 8 Outage probability for FUE

Fig. 6 illustrates that as the user offloaded to FBS with increasing bias value throughput of MUE is increases. We consider 20 femtocells. The femtocells are attract 60 users with increasing bias factor. Throughput is increases with decreasing macrocell users. Maximum femtocells are deployed in the CE region, so overall performance of CE users increases. Fig. 7 illustrates that as the femtocell users increases with increasing $12 \mathrm{~dB}$ bias value the throughput decreases up to acceptable limit. Although the rate of call blocking probability decreases with ensuring maximum per user capacity of femtocell users. However overall throughput of HetNets increases. Selection of optimal bias values for maximise per user capacity can be overcome by our proposed scheme. Fig. 8 illustrates the fact that the outage probability of the FFR-3 scheme is significantly smaller compared with without FFR scheme. It shows the importance of proper interference mitigation techniques for using biased femtocells. As FFR-3 reduce the interference thus biased cell association could be used with greater influence.

\section{CONCLUSION}

Multi-tier wireless networks employing macrocell and femtocell are considered to be the choice of network deployment in $4 \mathrm{G}$ and $5 \mathrm{G}$. We provided a solution for coping with heavy load by biased cell association. Proper interference mitigation and intelligent base station selection to offload user can ensure better QoS for users. Our work provides a way of achieving optimal per user capacity without blocking or dropping call. It also kept outage probability of femtocell users under acceptable range. Simulation results have shown the validity of our work.

\section{REFERENCE}

[1] A. S. Afolabi, E. Munkabat, C. Ohta, and H. Tamaki, "Fair Resource Partitioning between Cell-centre and Cell-edge of FFR-Based Multi-tier Wireless Access Networks," In Proceeding of Wireless Communications, networking and mobile computing, Sept. 2011, p.1-4.

[2] M. Z. Chowdhury, M. A. Hossain, S. Ahmed, and Y. M. Jang, "Radio Resource Management Based on Reused Frequency Allocation for Dynamic Channel Borrowing Scheme in Wireless Networks," Wireless Network, vol. 21, no. 8, pp.2593-2607, Nov. 2015.

[3] I. Guvenc, "Capacity and Fairness Analysis of Heterogeneous Networks with Range Expansion and Interference Coordination," IEEE Communication Letters, vol. 15, no. 10, pp. 1084-1087, Oct. 2011.

[4] Y. Wang, S. Chen, H. Ji, and H. Zhang, "Load-Aware Dynamic Biasing Cell Association in Small Cell Networks," In Proceeding of Mobile and Wireless Networking Symposium, June 2014, p. 2684-2689.

[5] T. Kudo and T. Ohtsuki, "Q-Learning Based Cell Selection for UE Outage Reduction in Heterogeneous Networks," In Proceeding of Vehicular Technology, Sept. 2014, p. 1-5.

[6] H. EISawy, E. Hossain and D. I. Kim, "HetNets with Cognitive Small Cells: User Offloading and Distributed Channel Access Techniques," Communication Magazine IEEE, vol. 51, no. 6, pp. 28-36, June 2013.

[7] P. Tian, H. Tian, J. Zhu, L. Chen and X. She, "An Adaptive Bias Configuration Strategy for Range Expansion in LTE-advanced Heterogeneous Networks," In Proceeding of Communication Technology and Application, Dec. 2010, p. 336-340.

[8] N. Saquib, E. Hossain, and D. I. Kim, "Fractional Frequency Reuse for Interference Management in LTE-Advanced HetNets," IEEE Transactions on Wireless Communications, vol. 20, no. 2, pp. 113-122, Apr. 2013.

[9] M. Z. Chowdhury and Y. M. Jang, "Handover Management in High Dense Femtocellular Networks," EURASIP Journal on Wireless Communications and Networking, pp. 1-21, Jan. 2013.

[10] H. S. Jo, Y. J. Sang, P. Xia, and J. G. Andrews, "Heterogeneous Cellular Networks with Flexible Cell Association: A Comprehensive Downlink SINR Analysis," IEEE Transactions Wireless Communications, vol. 11, no. 10, pp. 3484-3495, Aug. 2012.

[11] A. Morimoto, N. Miki, H. Ishii, and D. Nishikawa, "Investigation on Transmission Power Control in Heterogeneous Network Employing Cell Range Expansion for LTE-Advanced Uplink," in Proceedings of European Wireless Conference, Apr. 2012, p. 1-6. 